\begin{tabular}{|c|c|c|}
\hline & Int.J.Curr.Microbiol.App.Sci (2021) 10(10): 411-420 & \\
\hline EXCELLENT & $\begin{array}{l}\text { International Journal of Current Microbiology and Applied Sciences } \\
\text { ISSN: 2319-7706 Volume } 10 \text { Number } \mathbf{1 0}(\mathbf{2 0 2 1 )} \\
\text { Journal homepage: http://www.ijcmas.com }\end{array}$ & $\begin{array}{l}9 \\
39\end{array}$ \\
\hline PUBLISHERS & & www:ijcmas.com \\
\hline
\end{tabular}

\title{
An Economical Impact of Newcastle Disease Outbreaks in Various Commercial Broiler Chicken Farms During 2020-21 in Gujarat, India
}

\author{
V. A. Kalaria ${ }^{1}$, K. S. Prajapati ${ }^{1}$, B. B. Javia ${ }^{2}$, A. R. Bhadaniya ${ }^{1}$, D. T. Fefar ${ }^{1}$,
} A. A. Vagh $^{3}$, B. J. Trangadiya ${ }^{1}$, R. J. Padodara ${ }^{4}$, K. N. Mokaria ${ }^{1}$ and T. R. Kumbhani ${ }^{1}$

${ }^{1}$ Department of Veterinary Pathology, ${ }^{2}$ Department of Veterinary Microbiology,

${ }^{3}$ Department of Veterinary Medicine, ${ }^{4}$ Department of Veterinary Physiology and

Biochemistry, College of Veterinary Science \& Animal Husbandry, Kamdhenu University, Junagadh, Gujarat-362001, India

*Corresponding author

\section{A B S T R A C T}

Keywords

Newcastle disease, commercial broiler, economical impact

Article Info

Accepted:

15 September 2021

Available Online:

10 October 2021
Among the disease challenge, the Newcastle disease (ND) is the second most deadly viral infection and considered a very serious problem for poultry production in many countries. Outbreaks of the ND have a tremendous impact on the backyard as well as intensively reared commercial poultry farming and are considered as an unending agony of poultry farmers. During the study was observed that currently circulating NDV is viscerotropicvelogenic in nature and responsible for the outbreaks recorded in most of the vaccinated sampled commercial broiler farms which ultimately resulted in a huge economical impact on poultry farmers.

\section{Introduction}

The Indian poultry industry is one of the fastest-growing segments of the agricultural sector in India with around 8\% growth rate per annum. The constant efforts in development in poultry production have contributed to spectacular growth rates in egg $(4-6 \%$ per annum) and broiler production (8-10\% per annum) in India during the last 40 years (Chatterjee and Rajkumar, 2015). A striking feature of NDV strains and isolates is their ability to cause quite distinct signs and severity of the disease, even in the same host species. Depending upon the pathotype and susceptibility of the bird mortality rate varies from $0 \%$ to $100 \%$ (Nanthakumar et al., 2000). Outbreaks of ND may be devastating, with flock mortality approaching 100\% in fully susceptible chickens. Newcastle disease is considered economically influential diseasecausing huge production loss to the farmers of 
developing countries that export poultry or poultry products.

Losses resulting from trade restraints and high mortality is a major threat to the poultry industry (Narayanan et al., 2010).

During recent years, outbreaks of Newcastle disease were observed inspite of stringent and intensive vaccination policy against ND in many of the commercial broiler farms in Gujarat state, India. Few attempt has been made to estimate the economic losses due to ND among the commercial broiler chickens in the India.

Therefore, in this publication, we have calculated economic losses due to Newcastle disease outbreak occurred during 2019-21 in twenty-five commercial broiler farms located in three different geographical area of Gujarat state in India.

\section{Materials and Methods}

The information regarding the name and location of the farm, Farm ID No., Flock size, Flock mortality and Period of ND outbreaks was obtained directly from the farm owner at the time of necropsy examination or by making personal visits to the commercial broiler farms located at three different geographical areas of Gujarat where mortality was reported due to ND with or without regular vaccination program (Table-1).

The economical impact of ND in affected commercial broiler farms was calculated by using data recorded for epidemiological study as well as other information like overhead cost per bird per day (included labour, electricity and managemental expenses), current rate of day-old chicks, rate of per kg feed and selling rate of live bird/kg body weight, cost of ND vaccination, cost of routine medicine, biosecurity measures, and other vaccination for prevention of other diseases, cost of medicine during the course of ND etc. A newly modified economic model was developed and used to calculate the estimated economical impact of ND in all the twentyfive commercial broiler poultry farms included in the present study based on an earlier model of Williams (1999) and Khorajiya et al., (2018).

In viewing to better understanding and easiness of calculation, losses due to mortality (expenses on dead birds), expenses on live birds and income from selling of live birds were calculated separately and presented in respective tables.

Finally, the economical impact of ND in individual farms either in form of profit or losses was calculated by subtracting the total expenses on live birds and losses due to mortality (expanses on dead birds) from the income of selling live birds.

All kinds of costs or expenses and final profit or losses have been expressed in Indian currency rupees (₹) for a better realization in Indian perspectives.

\section{Economical losses calculated by}

\section{Losses due to mortality}

Losses due to mortality were calculated using the following formula.

$$
\begin{aligned}
& \mathrm{LM}=(\mathrm{NBD}) \times(\mathrm{VDOC}+\mathrm{CCFC}+\mathrm{OC}+\mathrm{CV} \\
& +\mathrm{CMVB})
\end{aligned}
$$

$\mathrm{LM}=$ Losses due to mortality

NBD $=$ Nos. of bird died

VDOC $=$ Value of day old chicks

CCFC $=$ Cost of cumulative feed consumed per dead bird 
$\mathrm{OC}=$ Overhead cost per dead bird

$\mathrm{CV}=$ Cost of ND vaccine per dead bird

CMVB = Cost of medicine, vaccine and biosecurity measures per dead bird for prevention of other diseases including medication during course of ND

\section{Expenditure on live birds}

$\mathrm{ELB}=(\mathrm{NBL}) \times(\mathrm{VDOC}+\mathrm{CCFC}+\mathrm{OC}+\mathrm{CV}$ + CMVB)

ELB $=$ Expenditure on live birds

$\mathrm{NBL}=$ Nos. of birds live

VDOC $=$ Value of day old chicks

CCFC $=$ Cost of cumulative feed consumed per live bird

$\mathrm{OC}=$ Overhead cost per live bird

$\mathrm{CV}=$ Cost of ND vaccine per live bird

CMVB = Cost of medicine, vaccine and biosecurity measures per live bird for prevention of other diseases including medication during course of ND

\section{Income from selling of live bird}

$\mathrm{ISLB}=(\mathrm{NBL}) \times(\mathrm{BWLB} \times \mathrm{SRLB})$

ISBL = Income from selling of live bird

$\mathrm{NBL}=$ Nos. of bird live at the time of selling

BWLB = Bodyweight per live bird at the time of selling

SRLB $=$ Selling rate per $\mathrm{kg}$ body weight of live bird at the time of selling
Economical impact of ND in various commercial broiler farms

$\mathrm{EI}=\mathrm{ISLB}-\mathrm{TE}$

$\mathrm{EI}=$ Economical impact

ISLB = Income from selling of live bird

$\mathrm{TE}=$ Total expenditure TE $=\mathrm{LM}+\mathrm{ELB}$

$\mathrm{LM}=$ Losses due to mortality

ELB $=$ Expenditure on live birds

\section{Results and Discussion}

Total losses due to mortality (expanses on dead birds) caused by ND and total expenditure on live birds in all the twenty-five commercial broiler farms was ranged between ₹ 17,864 to ₹ $2,66,080$ (Table-2) and ₹ $2,78,393$ to ₹ $17,86,745$ (Table-3) respectively. Whereas, income from the selling of live birds of individual farms was ranged between ₹ $1,60,255$ to ₹ $12,46,314$ (Table-4). The economical impact of ND in the individual farm in form of profit/loss in rupees as well as in percentage and also in form of profit/loss per bird in rupees is presented in Table-5. The profit/loss in percentage and profit/loss per bird in rupees was calculated from total profit/loss against total expenditure and initial flock size of individual farm respectively. As shown in Table-5, ₹ 74,290 and ₹ 94,514 profit was observed individually in only two farms.

The profit in form of percentages and ₹ / bird in these two farms was $14.23 \%$, ₹ 27.51 and $12.62 \%$, ₹ 23.63 respectively. The reason behind profit seen in these two farms was low mortality rate, the occurrence of disease at an early age lead to fewer expenses on dead birds, and higher body weight of birds at the time of selling and sold at market rate. 
Table.1 Name and location, Farm ID No., Flock size, flock mortality of commercial broiler farms affected with ND.

\begin{tabular}{|c|c|c|c|c|c|}
\hline $\begin{array}{l}\text { Sr. } \\
\text { No. }\end{array}$ & $\begin{array}{c}\text { Name and location of commercial broiler } \\
\text { poultry farms }\end{array}$ & Farm ID No. & $\begin{array}{c}\text { Flock Size } \\
\text { (No. of birds) }\end{array}$ & $\begin{array}{l}\text { Flock mortality } \\
\text { (No. of birds) }\end{array}$ & $\begin{array}{l}\text { Period of ND } \\
\text { outbreaks }\end{array}$ \\
\hline 1 & KGN Poultry Farm, Veraval, Gir-Somnath & GUJ/NDV/1 & 2200 & 350 & May - 2019 \\
\hline 2 & Afzal Poultry Farm, Mangrol, Junagadh & GUJ/NDV/2 & 2700 & 280 & May - 2019 \\
\hline 3 & Azad Poultry Farm, Dungarpur, Junagadh & GUJ/NDV/3 & 2500 & 530 & May-June - 2019 \\
\hline 4 & Patel Poultry Farm, Makhiyana, Junagadh & GUJ/NDV/4 & 4000 & 245 & May-June - 2019 \\
\hline 5 & Atari Poultry Farm, Patan, GirSomnath & GUJ/NDV/5 & 3100 & 455 & June- 2019 \\
\hline 6 & Ankur Poultry Farm, Panchasiya, Morbi & GUJ/NDV/6 & 7000 & 1208 & March - 2021 \\
\hline 7 & Irfan Poultry Farm, Valasan, Morbi & GUJ/NDV/7 & 5000 & 903 & March - 2021 \\
\hline 8 & Idrish Poultry Farm, Valasan, Morbi & GUJ/NDV/8 & 4000 & 960 & March - 2021 \\
\hline 9 & Noori Poultry Farm, Pipaliyaraj, Morbi & GUJ/NDV/9 & 4000 & 817 & March - 2021 \\
\hline 10 & Sabudin Poultry Farm, Pipaliyaraj, Morbi & GUJ/NDV/10 & 8000 & 880 & March - 2021 \\
\hline 11 & Samdani Poultry Farm, Pipaliyaraj, Morbi & GUJ/NDV/11 & 5000 & 919 & March - 2021 \\
\hline 12 & Sipai Poultry Farm, Kankot, Morbi & GUJ/NDV/12 & 6000 & 2130 & March - 2021 \\
\hline 13 & Yusuf Poultry Farm, Kankot, Morbi & GUJ/NDV/13 & 3500 & 840 & March - 2021 \\
\hline 14 & Gebi Poultry Farm, Tithva, Morbi & GUJ/NDV/14 & 4000 & 1890 & March - 2021 \\
\hline 15 & Sugar Poultry Farm, Tithva, Morbi & GUJ/NDV/15 & 6000 & 1130 & March - 2021 \\
\hline 16 & Sad Poultry Farm, Pratapgadh, Morbi & GUJ/NDV/16 & 3500 & 714 & March - 2021 \\
\hline 17 & Faizan Poultry Farm, Jodhpar, Morbi & GUJ/NDV/17 & 10000 & 1640 & March - 2021 \\
\hline 18 & Yusuf Poultry Farm, Chandrapur, Morbi & GUJ/NDV/18 & 4000 & 925 & March - 2021 \\
\hline 19 & Al-Amin Poultry Farm, Chandrapur, Morbi & GUJ/NDV/19 & 8000 & 1225 & March - 2021 \\
\hline 20 & Army Poultry Farm, Limbada, Morbi & GUJ/NDV/20 & 4000 & 1190 & March - 2021 \\
\hline 21 & Sarkar Poultry Farm, Limbada, Morbi & GUJ/NDV/21 & 8500 & 2080 & March - 2021 \\
\hline 22 & Taslim Poultry Farm, Rasikgadh, Morbi & GUJ/NDV/22 & 12000 & 1455 & March - 2021 \\
\hline 23 & Abdul Poultry Farm, Mahika, Morbi & GUJ/NDV/23 & 3500 & 760 & March - 2021 \\
\hline 24 & Haidar Poultry Farm, Paj, Morbi & GUJ/NDV/24 & 5000 & 535 & March - 2021 \\
\hline 25 & Ali Poultry Farm, Paj, Morbi & GUJ/NDV/25 & 7000 & 1060 & March - 2021 \\
\hline
\end{tabular}


Table.2 Total losses due to mortality in ND affected commercial broiler farms.

\begin{tabular}{|c|c|c|c|c|c|c|c|c|c|c|}
\hline $\begin{array}{l}\text { Sr. } \\
\text { No. }\end{array}$ & Farm ID No. & $\begin{array}{c}\text { Total } \\
\text { mortality } \\
\text { (No. of } \\
\text { bird } \\
\text { died) } \\
\text { (NBD) }\end{array}$ & $\begin{array}{l}\text { Value of } \\
\text { day old } \\
\text { chicks } \\
\text { (VDOC) } \\
\text { (₹) }\end{array}$ & $\begin{array}{c}\text { Cumulative } \\
\text { feed } \\
\text { consumed / } \\
\text { dead bird } \\
\text { (Kg) }\end{array}$ & $\begin{array}{c}\text { Rate } \\
\text { of } \\
\text { feed } \\
/ \text { kg } \\
\text { (₹) }\end{array}$ & $\begin{array}{l}\text { Cost of } \\
\text { cum. feed } \\
\text { consumed / } \\
\text { dead bird } \\
\text { (CCFC) } \\
\text { (₹) }\end{array}$ & $\begin{array}{c}\text { Overhead } \\
\text { cost / } \\
\text { dead bird } \\
@ 0.20 \\
\text { ₹/day } \\
\text { (OC) } \\
\text { (₹) }\end{array}$ & $\begin{array}{l}\text { Cost of } \\
\text { ND } \\
\text { vaccine } \\
\text { / dead } \\
\text { bird } \\
\text { (CV) } \\
\text { (₹) }\end{array}$ & $\begin{array}{c}\text { Cost of medicine, } \\
\text { vaccine and } \\
\text { biosecurity } \\
\text { measures/dead } \\
\text { bird } \\
\text { (CMVB) } \\
\text { (₹) }\end{array}$ & $\begin{array}{c}\text { Total losses due } \\
\text { to mortality } \\
\text { LM = NBD } \times \\
\text { (VDOC + } \\
\text { CCFC + OC + } \\
\text { CV + CMVB) } \\
\text { (₹) }\end{array}$ \\
\hline 1 & GUJ/NDV/1 & 350 & 42 & 1.54 & 35 & 53.90 & 5.00 & 0.30 & 4.00 & 36820 \\
\hline 2 & GUJ/NDV/2 & 280 & 42 & 0.44 & 35 & 15.40 & 2.60 & 0.80 & 3.00 & 17864 \\
\hline 3 & GUJ/NDV/3 & 530 & 42 & 1.47 & 35 & 51.45 & 5.00 & 0.00 & 3.50 & 54034 \\
\hline 4 & GUJ/NDV/4 & 245 & 42 & 0.86 & 35 & 30.10 & 3.60 & 0.80 & 3.50 & 19600 \\
\hline 5 & GUJ/NDV/5 & 455 & 41 & 1.58 & 35 & 55.30 & 5.00 & 0.15 & 4.00 & 47980 \\
\hline 6 & GUJ/NDV/6 & 1208 & 29 & 2.46 & 34 & 83.64 & 6.40 & 0.30 & 4.00 & 148995 \\
\hline 7 & GUJ/NDV/7 & 903 & 29 & 2.62 & 34 & 89.08 & 6.40 & 0.30 & 4.00 & 116288 \\
\hline 8 & GUJ/NDV/8 & 960 & 29 & 3.41 & 34 & 115.94 & 7.40 & 0.30 & 4.00 & 150374 \\
\hline 9 & GUJ/NDV/9 & 817 & 29 & 2.80 & 34 & 95.20 & 6.80 & 0.80 & 4.00 & 110949 \\
\hline 10 & GUJ/NDV/10 & 880 & 29 & 2.70 & 34 & 91.80 & 6.60 & 0.80 & 4.00 & 116336 \\
\hline 11 & GUJ/NDV/11 & 919 & 29 & 2.94 & 34 & 99.96 & 6.80 & 0.30 & 4.00 & 128715 \\
\hline 12 & GUJ/NDV/12 & 2130 & 29 & 2.53 & 34 & 86.02 & 6.40 & 0.00 & 3.50 & 266080 \\
\hline 13 & GUJ/NDV/13 & 840 & 29 & 2.86 & 34 & 97.24 & 6.80 & 0.80 & 4.00 & 115786 \\
\hline 14 & GUJ/NDV/14 & 1890 & 29 & 2.32 & 34 & 78.88 & 6.20 & 0.00 & 3.50 & 222226 \\
\hline 15 & GUJ/NDV/15 & 1130 & 29 & 2.34 & 34 & 79.56 & 6.00 & 0.80 & 4.00 & 134877 \\
\hline 16 & GUJ/NDV/16 & 714 & 25 & 3.41 & 34 & 115.94 & 7.60 & 0.30 & 4.00 & 109128 \\
\hline 17 & GUJ/NDV/17 & 1640 & 29 & 2.07 & 34 & 70.38 & 5.80 & 0.30 & 4.00 & 179547 \\
\hline 18 & GUJ/NDV/18 & 925 & 32 & 1.98 & 34 & 67.32 & 5.60 & 0.30 & 4.00 & 101029 \\
\hline 19 & GUJ/NDV/19 & 1225 & 29 & 3.26 & 34 & 110.84 & 7.20 & 0.30 & 4.00 & 185392 \\
\hline 20 & GUJ/NDV/20 & 1190 & 29 & 3.01 & 34 & 102.34 & 7.00 & 0.80 & 4.00 & 170337 \\
\hline 21 & GUJ/NDV/21 & 2080 & 29 & 2.39 & 34 & 81.26 & 6.20 & 0.80 & 4.00 & 252221 \\
\hline 22 & GUJ/NDV/22 & 1455 & 29 & 3.47 & 34 & 117.98 & 7.40 & 0.30 & 4.00 & 230879 \\
\hline 23 & GUJ/NDV/23 & 760 & 29 & 2.33 & 34 & 79.22 & 6.00 & 0.30 & 4.00 & 90075 \\
\hline 24 & GUJ/NDV/24 & 535 & 29 & 2.63 & 34 & 89.42 & 6.40 & 0.30 & 4.00 & 69079 \\
\hline 25 & GUJ/NDV/25 & 1060 & 29 & 2.09 & 34 & 71.06 & 5.80 & 0.80 & 4.00 & 117300 \\
\hline
\end{tabular}


Table.3 Expenditures on live birds in ND affected commercial broiler farms.

\begin{tabular}{|c|c|c|c|c|c|c|c|c|c|c|}
\hline $\begin{array}{l}\text { Sr. } \\
\text { No. }\end{array}$ & Farm ID No. & $\begin{array}{c}\text { No. of } \\
\text { bird live } \\
\text { (NBL) }\end{array}$ & $\begin{array}{l}\text { Value of } \\
\text { day old } \\
\text { chicks } \\
\text { (VDOC) } \\
\text { (₹) }\end{array}$ & $\begin{array}{c}\text { Cumulative } \\
\text { feed } \\
\text { consumed / } \\
\text { live bird } \\
\text { (Kg) }\end{array}$ & $\begin{array}{l}\text { Rate } \\
\text { of } \\
\text { feed } \\
/ \text { kg } \\
\text { (₹) }\end{array}$ & $\begin{array}{c}\text { Cost of } \\
\text { cumulative } \\
\text { feed } \\
\text { consumed / } \\
\text { live bird } \\
(\mathrm{CCFC})(₹)\end{array}$ & $\begin{array}{l}\text { Overhead } \\
\text { cost / live } \\
\text { bird @ } \\
0.20 \text { ₹/day } \\
\text { (OC) } \\
\text { (₹) }\end{array}$ & $\begin{array}{l}\text { Cost of } \\
\text { ND } \\
\text { vaccine / } \\
\text { live bird } \\
\text { (CV) } \\
\text { (₹) }\end{array}$ & $\begin{array}{c}\text { Cost of } \\
\text { medicine, } \\
\text { vaccine and } \\
\text { biosecurity } \\
\text { measures / live } \\
\text { bird (CMVB) } \\
\text { (₹) }\end{array}$ & $\begin{array}{c}\text { Expenditure on } \\
\text { live bird } \\
\text { ELB }=\text { NBL } \times \\
\text { (VDOC + CCFC } \\
+ \text { OC + CV + } \\
\text { CMVB) } \\
\text { (₹) }\end{array}$ \\
\hline 1 & GUJ/NDV/1 & 1850 & 42 & 4.22 & 35 & 147.70 & 8.40 & 0.30 & 4.50 & 375365 \\
\hline 2 & GUJ/NDV/2 & 2420 & 42 & 4.36 & 35 & 152.60 & 8.40 & 0.80 & 4.50 & 504086 \\
\hline 3 & GUJ/NDV/3 & 1970 & 42 & 4.41 & 35 & 154.35 & 9.00 & 0.00 & 4.00 & 412420 \\
\hline 4 & GUJ/NDV/4 & 3755 & 42 & 3.97 & 35 & 138.95 & 8.00 & 0.80 & 4.50 & 729409 \\
\hline 5 & GUJ/NDV/5 & 2645 & 41 & 4.48 & 35 & 156.80 & 8.80 & 0.15 & 4.50 & 558756 \\
\hline 6 & GUJ/NDV/6 & 5792 & 29 & 2.99 & 34 & 101.66 & 7.20 & 0.30 & 4.50 & 826287 \\
\hline 7 & GUJ/NDV/7 & 4097 & 29 & 3.01 & 34 & 102.34 & 7.00 & 0.30 & 4.50 & 586445 \\
\hline 8 & GUJ/NDV/8 & 3040 & 29 & 3.80 & 34 & 129.20 & 8.00 & 0.30 & 4.50 & 519840 \\
\hline 9 & GUJ/NDV/9 & 3183 & 29 & 3.38 & 34 & 114.92 & 7.60 & 0.80 & 4.50 & 499158 \\
\hline 10 & GUJ/NDV/10 & 7120 & 29 & 3.18 & 34 & 108.12 & 7.20 & 0.80 & 4.50 & 1065294 \\
\hline 11 & GUJ/NDV/11 & 4081 & 29 & 3.35 & 34 & 113.90 & 7.40 & 0.30 & 4.50 & 632963 \\
\hline 12 & GUJ/NDV/12 & 3870 & 29 & 2.83 & 34 & 96.22 & 6.80 & 0.00 & 4.00 & 526397 \\
\hline 13 & GUJ/NDV/13 & 2660 & 29 & 3.18 & 34 & 108.12 & 7.20 & 0.80 & 4.50 & 397989 \\
\hline 14 & GUJ/NDV/14 & 2110 & 29 & 2.71 & 34 & 92.14 & 6.80 & 0.00 & 4.00 & 278393 \\
\hline 15 & GUJ/NDV/15 & 4870 & 29 & 2.60 & 34 & 88.40 & 6.40 & 0.80 & 4.50 & 628717 \\
\hline 16 & GUJ/NDV/16 & 2786 & 25 & 3.97 & 34 & 134.98 & 8.40 & 0.30 & 4.50 & 482479 \\
\hline 17 & GUJ/NDV/17 & 8360 & 29 & 2.47 & 34 & 83.98 & 6.40 & 0.30 & 4.50 & 1038145 \\
\hline 18 & GUJ/NDV/18 & 3075 & 32 & 2.16 & 34 & 73.44 & 5.80 & 0.30 & 4.50 & 356823 \\
\hline 19 & GUJ/NDV/19 & 6775 & 29 & 3.66 & 34 & 124.44 & 7.80 & 0.30 & 4.50 & 1124921 \\
\hline 20 & GUJ/NDV/20 & 2810 & 29 & 3.32 & 34 & 112.88 & 7.40 & 0.80 & 4.50 & 434370 \\
\hline 21 & GUJ/NDV/21 & 6420 & 29 & 2.85 & 34 & 96.90 & 7.00 & 0.80 & 4.50 & 887244 \\
\hline 22 & GUJ/NDV/22 & 10545 & 29 & 3.76 & 34 & 127.84 & 7.80 & 0.30 & 4.50 & 1786745 \\
\hline 23 & GUJ/NDV/23 & 2740 & 29 & 2.58 & 34 & 87.72 & 6.40 & 0.30 & 4.50 & 350501 \\
\hline 24 & GUJ/NDV/24 & 4465 & 29 & 3.03 & 34 & 103.02 & 7.00 & 0.30 & 4.50 & 642156 \\
\hline 25 & GUJ/NDV/25 & 5940 & 29 & 2.64 & 34 & 89.76 & 6.80 & 0.80 & 4.50 & 777308 \\
\hline
\end{tabular}


Table.4 Income from selling of live birds of commercial broiler farms affected with ND.

\begin{tabular}{|c|c|c|c|c|c|}
\hline $\begin{array}{l}\text { Sr. } \\
\text { No. }\end{array}$ & Farm ID No. & $\begin{array}{l}\text { No. of bird } \\
\text { live } \\
(\text { NBL })\end{array}$ & $\begin{array}{c}\text { Body weight per live } \\
\text { bird } \\
\text { (BWLB) }(\mathrm{Kg})\end{array}$ & $\begin{array}{c}\text { Selling rate per kg body } \\
\text { weight of live bird (SRLB) } \\
\text { (₹) }\end{array}$ & $\begin{array}{c}\text { Income from selling of live bird } \\
\text { ISLB }=(\text { NBL }) \times(B W L B \times \\
\text { SRLB }) \\
\text { (₹) }\end{array}$ \\
\hline 1 & GUJ/NDV/1 & 1850 & 2.28 & 81 & 341658 \\
\hline 2 & GUJ/NDV/2 & 2420 & 2.54 & 97 & 596240 \\
\hline 3 & GUJ/NDV/3 & 1970 & 2.27 & 100 & 447190 \\
\hline 4 & GUJ/NDV/4 & 3755 & 2.34 & 96 & 843523 \\
\hline 5 & GUJ/NDV/5 & 2645 & 2.37 & 95 & 595522 \\
\hline 6 & GUJ/NDV/6 & 5792 & 1.76 & 51 & 519890 \\
\hline 7 & GUJ/NDV/7 & 4097 & 1.79 & 53 & 388682 \\
\hline 8 & GUJ/NDV/8 & 3040 & 2.08 & 51 & 322483 \\
\hline 9 & GUJ/NDV/9 & 3183 & 1.94 & 49 & 302576 \\
\hline 10 & GUJ/NDV/10 & 7120 & 1.90 & 49 & 662872 \\
\hline 11 & GUJ/NDV/11 & 4081 & 2.03 & 51 & 422506 \\
\hline 12 & GUJ/NDV/12 & 3870 & 1.73 & 42 & 281194 \\
\hline 13 & GUJ/NDV/13 & 2660 & 1.93 & 49 & 251556 \\
\hline 14 & GUJ/NDV/14 & 2110 & 1.55 & 49 & 160255 \\
\hline 15 & GUJ/NDV/15 & 4870 & 1.67 & 42 & 341582 \\
\hline 16 & GUJ/NDV/16 & 2786 & 2.14 & 42 & 250406 \\
\hline 17 & GUJ/NDV/17 & 8360 & 1.57 & 53 & 695636 \\
\hline 18 & GUJ/NDV/18 & 3075 & 1.48 & 53 & 241203 \\
\hline 19 & GUJ/NDV/19 & 6775 & 2.12 & 53 & 761239 \\
\hline 20 & GUJ/NDV/20 & 2810 & 2.00 & 53 & 297860 \\
\hline 21 & GUJ/NDV/21 & 6420 & 1.70 & 53 & 578442 \\
\hline 22 & GUJ/NDV/22 & 10545 & 2.23 & 53 & 1246314 \\
\hline 23 & GUJ/NDV/23 & 2740 & 1.65 & 53 & 239613 \\
\hline 24 & GUJ/NDV/24 & 4465 & 1.88 & 56 & 470075 \\
\hline 25 & GUJ/NDV/25 & 5940 & 1.61 & 53 & 506860 \\
\hline
\end{tabular}


Table.5 Economical impact of ND in various commercial broiler farms.

\begin{tabular}{|c|c|c|c|c|c|c|c|c|c|}
\hline $\begin{array}{l}\text { Sr. } \\
\text { No. }\end{array}$ & Farm ID No. & $\begin{array}{c}\text { Flock size } \\
\text { (No. of birds) }\end{array}$ & $\begin{array}{l}\text { Total losses } \\
\text { due to } \\
\text { mortality } \\
\text { (LM) } \\
\text { (₹) }\end{array}$ & $\begin{array}{l}\text { Expenditure } \\
\text { on live bird } \\
\text { (ELB) } \\
\text { (₹) }\end{array}$ & $\begin{array}{c}\text { Total } \\
\text { expenditure } \\
\text { TE = LM + } \\
\text { ELB } \\
\text { (₹) }\end{array}$ & $\begin{array}{l}\text { Income from } \\
\text { selling of live } \\
\text { bird(ISLB) } \\
\text { (₹) }\end{array}$ & $\begin{array}{c}\begin{array}{c}\text { Economical } \\
\text { impact }\end{array} \\
\text { EI = ISLB }- \text { TE } \\
\text { (₹) }\end{array}$ & $\begin{array}{c}\text { Profit } \\
\text { / loss } \\
(\%)\end{array}$ & $\begin{array}{l}\text { Profit / } \\
\text { loss per } \\
\text { bird } \\
\text { (₹) }\end{array}$ \\
\hline 1 & GUJ/NDV/1 & 2200 & 36820 & 375365 & 412185 & 341658 & -70527 & -17.11 & -32.06 \\
\hline 2 & GUJ/NDV/2 & 2700 & 17864 & 504086 & 521950 & 596240 & 74290 & 14.23 & 27.51 \\
\hline 3 & GUJ/NDV/3 & 2500 & 54034 & 412420 & 466454 & 447190 & -19264 & -4.13 & -7.71 \\
\hline 4 & GUJ/NDV/4 & 4000 & 19600 & 729409 & 749009 & 843523 & 94514 & 12.62 & 23.63 \\
\hline 5 & GUJ/NDV/5 & 3100 & 47980 & 558756 & 606736 & 595522 & -11214 & -1.85 & -3.62 \\
\hline 6 & GUJ/NDV/6 & 7000 & 148995 & 826287 & 975282 & 519890 & -455392 & -46.69 & -65.06 \\
\hline 7 & GUJ/NDV/7 & 5000 & 116288 & 586445 & 702733 & 388682 & -314051 & -44.69 & -62.81 \\
\hline 8 & GUJ/NDV/8 & 4000 & 150374 & 519840 & 670214 & 322483 & -347731 & -51.88 & -86.93 \\
\hline 9 & GUJ/NDV/9 & 4000 & 110949 & 499158 & 610107 & 302576 & -307531 & -50.41 & -76.88 \\
\hline 10 & GUJ/NDV/10 & 8000 & 116336 & 1065294 & 1181630 & 662872 & -518758 & -43.90 & -64.84 \\
\hline 11 & GUJ/NDV/11 & 5000 & 128715 & 632963 & 761678 & 422506 & -339172 & -44.53 & -67.83 \\
\hline 12 & GUJ/NDV/12 & 6000 & 266080 & 526397 & 792477 & 281194 & -511283 & -64.52 & -85.21 \\
\hline 13 & GUJ/NDV/13 & 3500 & 115786 & 397989 & 513775 & 251556 & -262219 & -51.04 & -74.92 \\
\hline 14 & GUJ/NDV/14 & 4000 & 222226 & 278393 & 500619 & 160255 & -340364 & -67.99 & -85.09 \\
\hline 15 & GUJ/NDV/15 & 6000 & 134877 & 628717 & 763594 & 341582 & -422012 & -55.27 & -70.34 \\
\hline 16 & GUJ/NDV/16 & 3500 & 109128 & 482479 & 591607 & 250406 & -341201 & -57.67 & -97.49 \\
\hline 17 & GUJ/NDV/17 & 10000 & 179547 & 1038145 & 1217692 & 695636 & -522056 & -42.87 & -52.21 \\
\hline 18 & GUJ/NDV/18 & 4000 & 101029 & 356823 & 457852 & 241203 & -216649 & -47.32 & -54.16 \\
\hline 19 & GUJ/NDV/19 & 8000 & 185392 & 1124921 & 1310313 & 761239 & -549074 & -41.90 & -68.63 \\
\hline 20 & GUJ/NDV/20 & 4000 & 170337 & 434370 & 604707 & 297860 & -306847 & -50.74 & -76.71 \\
\hline 21 & GUJ/NDV/21 & 8500 & 252221 & 887244 & 1139465 & 578442 & -561023 & -49.24 & -66.00 \\
\hline 22 & GUJ/NDV/22 & 12000 & 230879 & 1786745 & 2017624 & 1246314 & -771310 & -38.23 & -64.28 \\
\hline 23 & GUJ/NDV/23 & 3500 & 90075 & 350501 & 440576 & 239613 & -200963 & -45.61 & -57.42 \\
\hline 24 & GUJ/NDV/24 & 5000 & 69079 & 642156 & 711235 & 470075 & -241160 & -33.91 & -48.23 \\
\hline 25 & GUJ/NDV/25 & 7000 & 117300 & 777308 & 894608 & 506860 & -387748 & -43.34 & -55.39 \\
\hline
\end{tabular}


However, losses were evident in twenty-three farms in ranged of lowest losses ₹ 11,214 to highest losses ₹ 7,71,310 and overall total losses in twenty-three farms were ₹ 80,17,549. The losses in form of percentages and ₹ / bird in individual farms were in a range between 1.85 to $67.99 \%$ and ₹ 3.62 to ₹ 97.49 respectively. Among twenty-three farms, higher percentages of losses were observed in the range of 33.91 to $67.99 \%(20 / 25)$. The reason behind higher percentages of losses in these twenty farms was continuous high mortality, and the occurrence of disease around four to five week of flock age leads to more expenses on dead birds. Because of continuous heavy mortality, there was a compulsion to sell the flock at a cheap rate (₹ 42 to ₹ 53) even though the current open market rate of broiler chicken was in the range of ₹ 92 to ₹ 103 per $\mathrm{kg}$ live body weight of broiler bird during this period. In contrast to this twenty farm, in three farms in which mortality was under control, flock reared up to marketable age and sold at market rate. Hence, in these three farm percentages of losses was in the range of $1.85 \%$ to $17.11 \%$.

The economic losses seen in this study were concurrent to the study in India, a total of 13 flocks of 11 layer farms affected with ND and economic losses ₹ 37,19,223 was reported (Khorajiya et al., 2018). Newcastle disease in India led to heavy economic losses in commercial poultry farms and our study was consistent with the study of Munir et al., (2012), which stated that heavy economic losses to commercial poultry in Southeast Asia due to Newcastle disease outbreaks.

Similar results have been also observed and heavy economic losses of a total 200 million USD inspite of standard vaccination protocols and biosecurity measures among commercial poultry farms affected with Newcastle disease in Pakistan during 2011-2013 (Siddique et al., 2013). Antipas et al., (2012) observed 35 billion losses in the poultry industry with $55 \%$ mortality due to ND outbreaks in Chad. Mishra (1991) stated that in Nepal, Newcastle disease causes great economic loss by mortality and morbidity, estimated 75 million rupees per year.

The present study revealed that among twentyfive commercial broiler farms, twenty-three farms were highly affected due to Newcastle disease. Further, the outbreak of ND in these farms was solely responsible for ₹ 80,17,549 (₹ 8.01 million) economic losses. It gives a clear idea that once ND outbreak occurred in the farm it led to a very negative economic impact throughout the lifespan of the flock.

\section{Acknowledgments}

The authors express profound gratitude to Junagadh Agricultural University, Junagadh, Gujarat and Dean, College of Veterinary Science and Animal Husbandry, Kamdhenu University, Junagadh for providing the infrastructure facilities as well as financial help to carry out the research work.

\section{References}

Chatterjee, R. N. and Rajkumar, U. 2015. An overview of poultry production in India. Indian Journal of Animal Health, 54(2):89-108.

Narayanan, M.S., Parthiban, M., Sathiya, P. and Kumanan, K. 2010. Molecular detection of Newcastle disease virus using Flinders Tehnology AssociatesPCR. VeterinarskiArhiv, 80(1):51-60.

Nanthakumar, T., Kataria, R. S., Tiwari, A. K., Butchaiah, G. and Kataria, J. M. 2000. Pathotyping of Newcastle disease viruses by RT-PCR and restriction enzyme analysis. Veterinary Research Communications, 24(4):275286.

Antipas, B. B., Bidjeh, K. and Youssouf, M. 
L. 2012. Epidemiology of Newcastle disease and its economic impact in chad. European Journal of Experimental Biology, 2(6):2286-2292. Khorajiya, J. H., Joshi, B. P., Mathakiya, R. A., Prajapati, K. S. and Sipai, S. H. 2018. Economic Impact of GenotypeXIII Newcastle Disease Virus Infection on Commercial Vaccinated Layer Farms in India. International Journal of Livestock Research, 8(5):280-288.

Mishra U. 1991. Present Status of Poultry in Nepal. In: Newcastle Disease in Village Chickens, Control with Thermostable Oral Vaccines,
Proceedings of an international workshop of ACIAR, Kuala Lumpur, Malaysia. Pp. 163-166.

Munir, M., Zohari, S., Abbas, M. and Berg, M. 2012. Sequencing and analysis of the complete genome of Newcastle disease virus isolated from a commercial poultry farm in 2010. Archives of virology, 157(4):765-768.

Williams, R. B. 1999. A compartmentalised model for the estimation of the cost of coccidiosis to the world's chicken production industry. International Journal of Parasitology. 29(8): 12091229.

\section{How to cite this article:}

Kalaria, V. A., K. S. Prajapati, B. B. Javia, A. R. Bhadaniya, D. T. Fefar, A. A. Vagh, B. J. Trangadiya, R. J. Padodara, K. N. Mokaria and Kumbhani, T. R. 2021. An Economical Impact of Newcastle Disease Outbreaks in Various Commercial Broiler Chicken Farms During 202021 in Gujarat, India. Int.J.Curr.Microbiol.App.Sci. 10(10): 411-420.

doi: https://doi.org/10.20546/ijcmas.2021.1010.049 